\title{
Drug-Eluting Bead Transarterial Chemoembolization Combined with FOLFOX-Based Hepatic Arterial Infusion Chemotherapy for Large or Huge Hepatocellular Carcinoma
}

\author{
Jingjun Huang (iD) ${ }^{1, *}$ \\ Wensou Huang $\left(\mathrm{D}^{1}\right)^{1} *$ \\ Meixiao Zhan ${ }^{2, *}$ \\ Yongjian Guo' \\ Licong Liang' \\ Mingyue Cai ${ }^{1}$ \\ Liteng Lin' \\ Mingji $\mathrm{He}^{3}$ \\ Hui Lian (iD ${ }^{3}$ \\ Ligong Lu ${ }^{2}$ \\ Kangshun Zhu (iD)
}

'Department of Interventional Radiology, Minimally Invasive and Interventional Cancer Center, The Second Affiliated Hospital of Guangzhou Medical University, Guangzhou City, Guangdong Province, People's Republic of China;

${ }^{2}$ Zhuhai Interventional Medical Center, Zhuhai Precision Medical Center, Zhuhai People's Hospital, Zhuhai Hospital Affiliated with Jinan University, Zhuhai City, Guangdong Province, People's Republic of China; ${ }^{3}$ Department of Radiology, The Second Affiliated Hospital of Guangzhou Medical University, Guangzhou City, Guangdong Province, People's Republic of China

*These authors contributed equally to this work

Correspondence: Kangshun Zhu

Department of Interventional Radiology,

Minimally Invasive and Interventional Cancer

Center, The Second Affiliated Hospital of

Guangzhou Medical University, 250 East

Changgang Road, Guangzhou City, Guangdong,

51 0260, People's Republic of China

Tel +86-20-34l56205

Fax +86-20-34I53709

Email zhksh010@I63.com

Ligong Lu

Zhuhai Interventional Medical Center, Zhuhai

Precision Medical Center, Zhuhai People's

Hospital, Zhuhai Hospital Affiliated with Jinan

University, 79 Kangning Road, Zhuhai City,

Guangdong Province, 519000, People's Republic

of China

Tel +86 7-56-2222569

Fax +86 7-56-2162086

Email lu_ligong@I63.com
Purpose: To evaluate the safety and efficacy of drug-eluting bead transarterial chemoembolization (DEB-TACE) combined with oxaliplatin plus fluorouracil and leucovorin (FOLFOX)-based hepatic arterial infusion chemotherapy (D-TACE-HAIC) for unresectable large $(5.1-10 \mathrm{~cm})$ or huge $(>10 \mathrm{~cm})$ hepatocellular carcinoma $(\mathrm{HCC})$.

Methods: This retrospective study evaluated consecutive patients with unresectable large or huge HCC who underwent D-TACE-HAIC (D-TACE-HAIC group) or DEB-TACE (DEBTACE group) from January 2017 to December 2020. At imaging, tumor infiltrating appearance was classified into smooth tumor margin, non-smooth tumor margin, and macrovascular invasion. Adverse events, objective response rate (ORR), progression-free survival (PFS), and overall survival (OS) were compared between the two groups.

Results: A total of 133 patients (mean age, 53 years $\pm 12 ; 117$ men) were included: 69 underwent D-TACE-HAIC and 64 underwent DEB-TACE. The patients who underwent D-TACE-HAIC had higher ORR $(71.0 \%$ vs $53.1 \%$; $P=0.033)$, longer PFS (median, 9.3 vs 6.3 months; $P=0.005$ ), and better OS (median, 19.0 vs 14.0 months; $P=0.008$ ) than those who underwent DEB-TACE. In subgroup analysis, patients with non-smooth tumor margin (median, 20.8 vs 13.0 months; $P=0.031$ ) or macrovascular invasion (median, 15.0 vs 11.0 months; $P=0.015$ ) had significantly longer OS in D-TACE-HAIC group than in DEBTACE group; but in patients with smooth tumor margin, OS between the two groups was similar (median, 37.0 vs 35.0 months; $P=0.458$ ). DEB-TACE, non-smooth tumor margin, and macrovascular invasion were independent prognostic factors for poor OS in uni- and multivariable analyses. The incidence of grade $3 / 4$ adverse events was not statistically different between the two groups $(37.7 \%$ vs $28.1 \% ; P=0.242)$.

Conclusion: D-TACE-HAIC was tolerable and led to better OS than DEB-TACE in patients with large or huge HCC, especially in those with non-smooth tumor margin or macrovascular invasion.

Keywords: liver cancer, high tumor burden, chemoembolization, drug-eluting beads, hepatic arterial infusion chemotherapy, survival analysis

\section{Introduction}

The management of hepatocellular carcinomas (HCC) in patients with large $(5.1-10 \mathrm{~cm}$ in diameter) or huge ( $>10 \mathrm{~cm}$ in diameter) tumors who are not candidates for surgical resection remains a major challenge. ${ }^{1}$ The large or huge HCC was usually unresectable due to insufficient surgical margin, a residual liver volume estimated less than $30 \%$ after 
resection, or macrovascular invasion. ${ }^{2}$ The efficacy of conventional transarterial chemoembolization (c-TACE) using lipiodol mixed with chemotherapeutics on large or huge HCC is very limited, with poor objective response rate (ORR) of 16$29 \%$ and overall survival (OS) of only 6.5-9.1 months. ${ }^{4,5}$ Different from c-TACE, TACE using drug-eluting beads (DEB-TACE) can increase the intensity and duration of tumor ischemic necrosis and deliver large amounts of chemotherapeutics to the tumor in a controlled and sustained manner. ${ }^{6,7}$ For large or huge HCC, DEB-TACE may lead to higher $\mathrm{ORR}^{3,8,9}$ and longer time to progression ${ }^{8,10}$ with an improved tolerability ${ }^{9,11}$ when compared with c-TACE.

However, the OS of DEB-TACE in patients with large or huge HCC is still not satisfactory, with a median OS of only 13.0-16.0 months. ${ }^{12,13}$ One reason is that complete embolization of HCC with large tumor burden by one single procedure could cause serious embolization-related adverse events (AEs), such as serious post-embolization syndrome, liver/renal dysfunction, biliary injury, and liver abscess, ${ }^{14,15}$ so DEB-TACE with multiple procedures is recommended for large or huge HCC. ${ }^{16}$ However, the residual arterial supply following each embolization may contribute to the progression of residual tumor. ${ }^{17}$ Moreover, large or huge HCCs usually have multiple collateral arterial supplies, ${ }^{18}$ vascular invasion, ${ }^{19}$ or arteriovenous fistula, ${ }^{9}$ which directly affect the efficacy of DEB-TACE.

Recently, several studies have shown that oxaliplatin plus fluorouracil and leucovorin (FOLFOX)-based hepatic arterial infusion chemotherapy (HAIC) could offer better treatment responses and survival benefits than sorafenib or c-TACE for large or huge HCC. ${ }^{20,21}$ HAIC directly delivers high concentration of chemotherapeutics into the tumor to enhance drug exposure, which avoids embolization-related AEs. However, the efficacy of HAIC majorly relies on the chemosensitivity of the tumor and drugs lodged in the tumor for a short period of time and wash out quickly. ${ }^{7}$ Different from HAIC, DEB-TACE can embolize multiple tumor-feeding arteries and rapidly lead to substantial ischemic tumor necrosis, and meanwhile significantly prolongs the contact time between cancer cells and chemotherapeutics. ${ }^{6,7,22}$ Thus, it seems that the combination of DEB-TACE and HAIC (D-TACE-HAIC) could make up the deficiency of DEB-TACE and HAIC to yield an enhanced local anti-tumor effect and mild AEs, especially in HCC with large tumor burden. Therefore, this study aimed to compare the safety and efficacy of
D-TACE-HAIC with DEB-TACE in patients with large or huge HCC.

\section{Materials and Methods Patient Selection}

We reviewed the electronic medical records of consecutive patients with large $(5.1-10 \mathrm{~cm})$ or huge $(>10 \mathrm{~cm})$ unresectable HCC who were treated with D-TACE-HAIC (D-TACE-HAIC group) or DEB-TACE alone (DEBTACE group) from January 2017 to December 2020 at our institution. The DEB-TACE combined with HAIC was approved by the institutional review board of the Second Affiliated Hospital of Guangzhou Medical University before being performed in clinical practice. Before these patients underwent initial DEB-TACE, the attending physician (K.Z., W.H., or Y.G.) recommended they choose DEB-TACE alone or DEB-TACE combined with HAIC. All patients were informed that DEB-TACE combined with HAIC was an alternative treatment, and informed of the potential toxicity. Final treatment decisions were made by the patients. This retrospective study was approved by the institutional review board and conducted in accordance with the Declaration of Helsinki. Written informed consent was obtained from every patient prior to treatment.

HCC was diagnosed according to the European Association for the Study of Liver and American Association for the study of Liver Disease guidelines. ${ }^{23}$ The inclusion criteria for the study population were as follows: 1) the maximum lesion accessed larger than $5 \mathrm{~cm}$ on dynamic CT or MR images obtained within 7 days before treatment, 2) age between 18 and 75 years, 3) Eastern Cooperative Oncology Group performance status of 0 or 1,4) Child-Pugh class A liver disease, and 5) adequate organ function, with hemoglobin $\geq 8.5 \mathrm{~g} / \mathrm{dL}$, white blood cell count $\geq 3.0 \times 10^{9} / \mathrm{L}$, neutrophil count $\geq 1.5 \times 10^{9} / \mathrm{L}$, platelet count $\geq 75 \times 10^{9} / \mathrm{L}$, aspartate transaminase and alanine transaminase $\leq 5 \times$ upper limit of the normal, and creatinine clearance rate of $\leq 1.5 \times$ upper limit of the normal. Patients were excluded from this study if they 1) had tumor invasion in bilateral first-order portal vein branch, the main trunk of portal vein, or inferior vena cava; 2) had evidence of extrahepatic metastasis before treatment; 3) had previously undergone sorafenib or lenvatinib therapy, systemic chemotherapy, HAIC, or TACE; 4) currently had or had a history of malignant tumors in addition to HCC; 5) had severe medical comorbidities including severe cardiopulmonary dysfunction and 
coagulation disorders (international normalized ratio $\geq 1.5$ ); or 6 ) had a follow-up less than 3 months.

\section{Imaging Assessments of $\mathrm{HCC}$ Characteristics}

All the dynamic CT or MR imaging analyses, including tumor size, tumor number, tumor margin, macrovascular invasion, and treatment response, were performed independently by two diagnostic radiologists who were blinded to treatment allocation and clinical information (M.H. and H.L., 21 and 18 years of experience in abdominal imaging, respectively). When there was any ambiguity, the final determination was made by consensus.

Tumor infiltrating appearance at imaging was classified as smooth tumor margin, non-smooth tumor margin, and macrovascular invasion. Macrovascular invasion was defined as the presence of tumor thrombus in the first- or lower-order portal vein branch or left, middle, or right hepatic veins. For tumor without macrovascular invasion, tumor margins on equilibrium phase images were divided into two categories according to the previous studies ${ }^{24,25}$ : smooth tumor margin, defined as a nodular tumor with a smooth tumor-normal liver interface in all axial, coronal, and sagittal imaging planes; and non-smooth tumor margin, defined as a nonnodular tumor in any imaging planes, including focal or crescent extranodular extension protruding into the nontumor parenchyma, multinodular confluence appearance, and focal infiltrative margin.

\section{Procedures}

Patients in both groups received the same procedure of DEB-TACE, performed by K.Z., W.H., or Y.G. (30, 12, and 10 years of experience performing TACE, respectively). Before begining the procedure, we prepared one vial of CalliSpheres ${ }^{\circledR}$ beads (Jiangsu Hengrui Medicine Co. Ltd., Jiangsu, P.R. China) or DC bead ${ }^{\circledR}$ (Biocompatibles, Farnham, Surrey, UK) with diameters of $100-300 \mu \mathrm{m}$ by extracting the supernatant, and then mixing the beads and the solution of $60 \mathrm{mg}$ pirarubicin (ShenZhen Main Luck Pharmaceuticals Inc., ShenZhen, P. R. China) for loading drugs. After loading drugs, all supernatant was extracted, and an equal quantity of nonionic contrast diluted with saline was added to form $30 \mathrm{~mL}$ suspension for injection. Before embolization, the location of tumor-feeding arteries was determined by digitalsubtraction angiography of celiac trunk, superior mesenteric artery, and phrenic artery, using a 5-F catheter (Cook,
Bloomington, Indiana) or a 2.8-F microcatheter (Renegade Hi-Flo Straight, Boston Scientific, Natick, MA; Progreat, Terumo, Tokyo, Japan). During the embolization, a microcatheter was inserted as super-selectively as possible into tumor-feeding arteries, and suspension of DEB was injected at a rate of $1-3 \mathrm{~mL} / \mathrm{min}$. In the case of significant arterioportal or hepatic venous shunting, embolization of the shunt with polyvinyl alcohol was performed before proceeding with DEB administration. If a tumor blush remained following one vial of DEB, 1-2 vials of 300-500 $\mu \mathrm{m}$ Embosphere ${ }^{\circledR}$ beads (Biosphere Medical, Roissy En, France) were injected to reduce blood flow. To reduce the incidences of post-embolization syndrome and liver toxicity, an embolization end point with arterial flow stasis was not achieved in the first procedure, but achieved in the second or third procedure according to tumor burden and follow-up imaging. ${ }^{16}$

In the D-TACE-HAIC group, after chemoembolization, the microcatheter was reserved at the proper/left/right hepatic artery. After the patient returned to the ward, the following FOLFOX-based regime was intra-arterially administered through the microcatheter: oxaliplatin, $85 \mathrm{mg} / \mathrm{m}^{2}$ infusion for 2 hours; leucovorin, $400 \mathrm{mg} / \mathrm{m}^{2}$ infusion for 2 hours; and 5-FU, $400 \mathrm{mg} / \mathrm{m}^{2}$ bolus infusion and then $2400 \mathrm{mg} / \mathrm{m}^{2}$ continuous infusion for 46 hours. For patients who encountered vomiting during or after the infusion, tropisetron $5 \mathrm{mg}$ or palonosetron $0.25 \mathrm{mg}$ was used by intravenous injection.

\section{Follow-Up and Repeated Procedure}

Follow-up of the patients was conducted at a 4-week interval after previous treatment. Patients with intrahepatic residual viable tumor on follow-up $\mathrm{CT}$ or MR imaging underwent repeated D-TACE-HAIC or DEB-TACE. Treatment was discontinued in cases of failure to achieve objective response in the targeted tumor after at least two procedures. The emergence of new intrahepatic lesion remoted from the targeted tumor, although representing tumor progression, did not contraindicate further treatment with D-TACE-HAIC or DEB-TACE. For the patients with emergence of new intrahepatic lesion, D-TACE-HAIC or DEB-TACE would be performed for the residual viable primary tumor and the new intrahepatic lesion, but the treatment would be discontinued if it failed to achieve objective response. In cases of clinical or functional deterioration, treatment was discontinued in patients who have clinical progression to ECOG performance status $>2$ or who experience evolution to sustained hepatic 
decompensation or intolerable toxicity. ${ }^{16}$ Patients with tumor progression might receive sorafenib/lenvatinib and/ or regional therapies, ie, TACE, microwave ablation, radiofrequency ablation, or Iodine 125 seed implantation, according to the attending physicians' consensus.

\section{Outcomes}

Adverse events related to treatment that occurred within 4 weeks after procedure were recorded, according to National Cancer Institute Common Terminology Criteria for Adverse Events, version 5.0. ${ }^{26}$

The best observed treatment responses were assessed based on the dynamic CT or MR imaging, according to the modified Response Evaluation Criteria in Solid Tumors. ${ }^{27}$ ORR was defined as the incidence of complete response and partial response. Disease control rate (DCR) was defined as the incidence of complete response, partial response, and stable disease.

Progression-free survival (PFS) was defined as the time from the initial procedure until the date when tumor progression or death was confirmed or the last follow-up in censored data. OS was defined as the time from the initial procedure until death or the last follow-up in censored data.

\section{Statistical Analyses}

The Pearson $\chi 2$ test, correction of continuity, or Fisher's exact test was used to compare the categorical data of baseline patient characteristics, adverse events, and treatment responses between the two groups, as appropriate. The Mann-Whitney $U$-test was used to compare the quantitative data of baseline patient characteristics between the two groups. Curves of OS and PFS were determined by the Kaplan-Meier method, and the Log rank test was used for comparisons. Univariate analyses and multivariate analyses of prognostic factors for OS and PFS were performed with Cox proportional hazard regression models. Variables with a $P$ value $<0.10$ in the univariate analysis were included into the multivariate analysis. All tests were 2-sided, and $P<0.05$ was considered statistically

\begin{tabular}{|c|c|c|c|}
\hline & \multicolumn{2}{|c|}{$\begin{array}{l}\text { Unresectable large or huge HCC treated with } \\
\text { D-TACE-HAIC or DEB-TACE }(01 / 2017-12 / 2020) \\
\text { Assessed for eligibility }(\mathrm{n}=164)\end{array}$} & \\
\hline \multicolumn{2}{|c|}{$\begin{array}{l}\text { HCC treated with D-TACE-HAIC } \\
\qquad(\mathrm{n}=88)\end{array}$} & \multicolumn{2}{|c|}{$\begin{array}{l}\text { HCC treated with DEB-TACE } \\
\qquad(\mathrm{n}=76)\end{array}$} \\
\hline $\begin{array}{l}\text { Excluded }(\mathrm{n}=19) \\
\text { - Main trunk of portal vein or } \\
\text { tumor thrombus }(\mathrm{n}=2) \\
\text { - Extrahepatic metastasis }(\mathrm{n}= \\
\text { - Previous TACE }(\mathrm{n}=6) \\
\text { - Previous sorafenib or lenvati } \\
=4) \\
\text { - Missing data of baseline CT } \\
\text { - Follow up less than three mo }\end{array}$ & $\begin{array}{l}\text { vena cava } \\
\text { 4) } \\
\text { inib therapy ( } \mathrm{n} \\
\text { or MR }(\mathrm{n}=1) \\
\text { nths }(\mathrm{n}=2)\end{array}$ & $\begin{array}{l}\text { Excluded }(\mathrm{n}= \\
\text { - Main trunk } \\
(\mathrm{n}=1) \\
\text { - Previous TA } \\
\text { - Previous so } \\
\text { - Other malig } \\
\text { - Medical cor } \\
\text { - Follow up l }\end{array}$ & $\begin{array}{l}\text { 2) } \\
\text { portal vein tumor thrombus } \\
\text { E or HAIC }(n=5) \\
\text { enib therapy }(n=3) \\
\text { nt tumor }(n=1) \\
\text { rbidities }(n=1) \\
\text { than three months }(n=1)\end{array}$ \\
\hline $\begin{array}{l}\text { Large or huge HCC treated wi } \\
\qquad(n=69)\end{array}$ & th D-TACE-HAIC & Large or huge $\mathrm{H}$ & $\begin{array}{l}\text { C treated with DEB-TACE } \\
(\mathrm{n}=64)\end{array}$ \\
\hline
\end{tabular}

Figure I Flow diagram shows exclusion in patients with large or huge hepatocellular carcinoma (HCC).

Abbreviations: D-TACE-HAIC, drug-eluting bead transarterial chemoembolization combined with hepatic arterial infusion chemotherapy; DEB-TACE, drug-eluting bead transarterial chemoembolization; HAIC, hepatic arterial infusion chemotherapy; c-TACE, conventional transarterial chemoembolization. 
Table I Baseline Patient Characteristics

\begin{tabular}{|c|c|c|c|}
\hline Characteristic & $\begin{array}{l}\text { D-TACE- } \\
\text { HAIC } \\
\text { Group (N = } \\
69)\end{array}$ & $\begin{array}{l}\text { DEB- } \\
\text { TACE } \\
\text { Group }(\mathbf{N} \\
=64)\end{array}$ & $P$ value \\
\hline $\begin{array}{l}\text { Sex } \\
\qquad \text { Male } \\
\text { Female }\end{array}$ & $\begin{array}{l}62(89.9) \\
7(10.1)\end{array}$ & $\begin{array}{l}55(85.9) \\
9(14.1)\end{array}$ & 0.488 \\
\hline $\begin{array}{l}\text { Age (years), Median } \\
\text { (IQR) } \\
\quad \leq 60 \\
>60\end{array}$ & $\begin{array}{l}55(44-62) \\
51(73.9) \\
18(26.1)\end{array}$ & $\begin{array}{l}51(42-62) \\
45(70.3) \\
19(29.7)\end{array}$ & $\begin{array}{l}0.652 \\
0.643\end{array}$ \\
\hline $\begin{array}{l}\text { BCLC stage } \\
\text { A } \\
\text { B } \\
\text { C }\end{array}$ & $\begin{array}{l}4(5.8) \\
26(37.7) \\
39(56.5)\end{array}$ & $\begin{array}{l}7(10.9) \\
25(39.1) \\
32(50.0)\end{array}$ & 0.511 \\
\hline $\begin{array}{l}\text { ECOG score } \\
\qquad \begin{array}{c}0 \\
1\end{array}\end{array}$ & $\begin{array}{l}55(79.7) \\
14(20.3)\end{array}$ & $\begin{array}{l}52(81.3) \\
12(18.8)\end{array}$ & 0.823 \\
\hline $\begin{array}{l}\text { Tumor size }(\mathrm{cm}) \text {, Median } \\
\text { (range) } \\
5.1-10 \\
>10\end{array}$ & $\begin{array}{l}10.4(5.1- \\
22.0) \\
34(49.3) \\
35(50.7)\end{array}$ & $\begin{array}{l}9.7(5.1- \\
20.0) \\
36(56.3) \\
28(43.8)\end{array}$ & $\begin{array}{l}0.662 \\
0.421\end{array}$ \\
\hline $\begin{array}{l}\text { Number of intrahepatic } \\
\text { tumors } \\
\qquad \begin{array}{l}\leq 3 \\
>3\end{array}\end{array}$ & $\begin{array}{l}39(56.5) \\
30(43.5)\end{array}$ & $\begin{array}{l}41(64.1) \\
23(35.9)\end{array}$ & 0.375 \\
\hline $\begin{array}{l}\text { Portal vein invasion } \\
\text { No } \\
\text { Second- or lower- } \\
\text { order branch } \\
\text { First-order branch }\end{array}$ & $\begin{array}{l}35(50.7) \\
17(24.6) \\
17(24.6)\end{array}$ & $\begin{array}{l}34(53.1) \\
13(20.3) \\
17(26.6)\end{array}$ & 0.835 \\
\hline $\begin{array}{l}\text { Tumor infiltrating } \\
\text { appearance } \\
\text { Smooth tumor margin } \\
\text { Non-smooth tumor } \\
\text { margin } \\
\text { Macrovascular invasion }\end{array}$ & $\begin{array}{l}17(24.6) \\
18(26.1) \\
34(49.3)\end{array}$ & $\begin{array}{l}16(25.0) \\
18(28.1) \\
30(46.7)\end{array}$ & 0.955 \\
\hline $\begin{array}{l}\text { Positive for HBsAg } \\
\text { No } \\
\text { Yes }\end{array}$ & $\begin{array}{l}4(5.8) \\
65(94.2)\end{array}$ & $\begin{array}{l}6(9.4) \\
58(90.6)\end{array}$ & 0.651 \\
\hline $\begin{array}{l}\alpha \text {-Fetoprotein }(\mathrm{ng} / \mathrm{mL}) \text {, } \\
\text { Median }(\mathrm{IQR}) \\
\quad \leq 400 \\
\quad>400\end{array}$ & $\begin{array}{l}305(17- \\
18,549) \\
36(52.2) \\
33(47.8)\end{array}$ & $\begin{array}{l}117(11- \\
1478) \\
39(60.9) \\
25(39.1)\end{array}$ & $\begin{array}{l}0.435 \\
0.309\end{array}$ \\
\hline $\begin{array}{l}\text { Total bilirubin }(\mu \mathrm{mol} / \mathrm{L}) \text {, } \\
\text { Median (IQR) } \\
\quad \leq 22\end{array}$ & $\begin{array}{l}15.6(10.3- \\
21.2) \\
54(78.3)\end{array}$ & $\begin{array}{l}15.2(10.4- \\
20.3) \\
50(78.1)\end{array}$ & $\begin{array}{l}0.662 \\
0.985\end{array}$ \\
\hline
\end{tabular}

(Continued)
Table I (Continued).

\begin{tabular}{|c|c|c|c|}
\hline Characteristic & $\begin{array}{l}\text { D-TACE- } \\
\text { HAIC } \\
\text { Group (N = } \\
69)\end{array}$ & $\begin{array}{l}\text { DEB- } \\
\text { TACE } \\
\text { Group (N } \\
=64)\end{array}$ & $P$ value \\
\hline$>22$ & $15(21.7)$ & 14 (21.9) & \\
\hline $\begin{array}{l}\text { Albumin }(\mathrm{g} / \mathrm{dL}) \text {, Median } \\
\begin{array}{l}\text { (IQR) } \\
\quad \geq 35 \\
\quad<35\end{array}\end{array}$ & $\begin{array}{l}36.6(33.0- \\
40.0) \\
45(65.2) \\
24(34.8)\end{array}$ & $\begin{array}{l}37.7(34.5- \\
40.2) \\
46(71.9) \\
18(28.1)\end{array}$ & $\begin{array}{l}0.440 \\
0.409\end{array}$ \\
\hline $\begin{array}{l}\text { Platelet }\left(10^{9} / \mathrm{L}\right), \text { Median } \\
\text { (IQR) } \\
\quad \geq 100 \\
\quad<100\end{array}$ & $\begin{array}{l}198(140- \\
267) \\
65(85.5) \\
4(5.8)\end{array}$ & $\begin{array}{l}189(134- \\
236) \\
55(85.9) \\
9(14.1)\end{array}$ & $\begin{array}{l}0.662 \\
0.109\end{array}$ \\
\hline $\begin{array}{l}\text { Post treatment }^{\mathrm{a}} \\
\text { Locoregional therapy } \\
\text { Sorafenib/lenvatinib } \\
\text { Combination of } \\
\text { sorafenib/lenvatinib and } \\
\text { locoregional therapy } \\
\text { Best supportive care }\end{array}$ & $\begin{array}{l}\mathrm{n}=46 \\
\text { II (23.9) } \\
\text { I5 (32.6) } \\
19(41.3) \\
\text { I }(2.2)\end{array}$ & $\begin{array}{l}n=53 \\
9(17.0) \\
21(39.6) \\
19(35.8) \\
4(7.5)\end{array}$ & 0.451 \\
\hline
\end{tabular}

Notes: Except where indicated, data are numbers of patients, and data in parentheses are percentages. ${ }^{a}$ Only for patients who failed the treatment of D-TACE-HAIC or DEB-TACE $(n=46$ in the D-TACE-HAIC group and $n=53$ in the DEB-TACE group).

Abbreviations: BCLC, Barcelona Clinic Liver Cancer; ECOG, Eastern Cooperative Oncology Group; D-TACE-HAIC, drug-eluting bead transarterial chemoembolization combined with hepatic arterial infusion chemotherapy; DEB-TACE, drug-eluting bead transarterial chemoembolization; IQR, interquartile range; $\mathrm{HBsAg}$, hepatitis B surface antigen.

significant. All statistical analyses were performed using SPSS Statistics, version 22.0 (SPSS, Chicago, IL).

\section{Results}

\section{Study Population}

A total of 164 patients with unresectable large or huge HCC who underwent D-TACE-HAIC or DEB-TACE were assessed for eligibility during the study period. Thirty-one patients were excluded because they met the exclusion criteria (Figure 1). Finally, 133 patients were included in this study (D-TACE-HAIC group, $\mathrm{n}=69$; DEB-TACE group, $n=64)$. The baseline characteristics between the two groups were not significantly different (Table 1). Huge HCC had a higher proportion of a non-smooth tumor margin or macrovascular invasion than large HCC $(85.7 \%$ [54/ 63] vs $65.7 \%$ [46/70], $P=0.008)$. Among 33 patients with a smooth tumor margin, $72.7 \%(24 / 33)$ had a tumor $5.1-$ $10 \mathrm{~cm}$ but only $27.3 \%(9 / 33)$ had a tumor $>10 \mathrm{~cm}$. 
Table 2 Adverse Events in the Two Groups

\begin{tabular}{|c|c|c|c|c|c|c|}
\hline \multirow[t]{2}{*}{ Adverse Events } & \multicolumn{3}{|c|}{ Any Grade } & \multicolumn{3}{|c|}{ Grade 3 or 4} \\
\hline & $\begin{array}{l}\text { D-TACE-HAIC } \\
\text { Group }(\mathrm{N}=69)\end{array}$ & $\begin{array}{l}\text { DEB-TACE } \\
\text { Group }(\mathrm{N}=64)\end{array}$ & $P$ value & $\begin{array}{l}\text { D-TACE-HAIC } \\
\text { Group }(\mathrm{N}=69)\end{array}$ & $\begin{array}{l}\text { DEB-TACE } \\
\text { Group }(\mathrm{N}=64)\end{array}$ & $P$ value \\
\hline Overall incidence & $65(94.2)$ & $57(89.1)$ & 0.282 & $26(37.7)$ & $18(28.1)$ & 0.242 \\
\hline \multicolumn{7}{|l|}{$\begin{array}{l}\text { Post-embolization } \\
\text { syndrome }\end{array}$} \\
\hline Fever & 41 (59.4) & $36(56.3)$ & 0.711 & $5(7.2)$ & $3(4.7)$ & 0.799 \\
\hline Abdominal pain & $42(60.9)$ & $30(46.9)$ & 0.106 & $13(18.8)$ & $6(9.4)$ & 0.119 \\
\hline Nausea/vomiting & $24(34.8)$ & $12(18.8)$ & 0.038 & $6(8.7)$ & $2(3.1)$ & 0.177 \\
\hline \multicolumn{7}{|l|}{ Liver dysfunction } \\
\hline Hypoalbuminemia & II (I5.9) & $5(7.8)$ & 0.150 & $2(2.9)$ & 0 & 0.497 \\
\hline Hyperbilirubinemia & $12(17.4)$ & $9(14.1)$ & 0.599 & $4(5.8)$ & I (I.6) & 0.408 \\
\hline ALT elevation & $22(31.9)$ & $13(20.3)$ & 0.130 & $6(8.7)$ & $4(6.3)$ & 0.837 \\
\hline AST elevation & $12(17.4)$ & $10(15.6)$ & 0.784 & $4(5.8)$ & $3(4.7)$ & $>0.999$ \\
\hline \multicolumn{7}{|l|}{ Hematologic toxicity } \\
\hline Anemia & $17(24.6)$ & $10(15.6)$ & 0.197 & $6(8.7)$ & $2(3.1)$ & 0.325 \\
\hline Leukopenia & $13(18.8)$ & $5(7.8)$ & 0.063 & $6(8.7)$ & $\mathrm{I}(\mathrm{I} .6)$ & 0.146 \\
\hline Neutropenia & $14(20.3)$ & $5(7.8)$ & 0.040 & $5(7.2)$ & $2(3.1)$ & 0.500 \\
\hline Thrombocytopenia & $20(29.0)$ & $8(12.5)$ & 0.020 & $8(11.6)$ & $2(3.1)$ & 0.128 \\
\hline Sensory neuropathy & $6(8.7)$ & 0 & 0.016 & 0 & 0 & $\ldots$ \\
\hline Diarrhea & $3(4.3)$ & $4(6.3)$ & 0.624 & 0 & 0 & $\ldots$ \\
\hline Fatigue & $15(21.7)$ & II (I7.2) & 0.508 & $5(7.2)$ & $3(4.7)$ & 0.799 \\
\hline Creatinine increase & $\mathrm{I}(1.6)$ & 0 & $0.48 \mathrm{I}$ & 0 & 0 & $\ldots$ \\
\hline Acute heart failure & 0 & $\mathrm{I}(\mathrm{I} .6)$ & $0.48 I$ & 0 & $\mathrm{I}(\mathrm{I} .6)$ & 0.481 \\
\hline Liver abscess & $2(2.9)$ & $3(4.7)$ & 0.932 & $2(2.9)$ & $3(4.7)$ & 0.932 \\
\hline $\begin{array}{l}\text { Segmental bile duct } \\
\text { dilatation }\end{array}$ & $3(4.3)$ & $5(7.8)$ & 0.635 & 0 & 0 & $\ldots$ \\
\hline Cholecystitis & $4(5.8)$ & $4(6.3)$ & $>0.999$ & $\mathrm{I}(\mathrm{I} .6)$ & 0 & $0.48 I$ \\
\hline Ascites/ Pleural effusion & $4(5.8)$ & $2(3.1)$ & 0.746 & 0 & $\mathrm{I}(\mathrm{I} .6)$ & 0.481 \\
\hline $\begin{array}{l}\text { Gastrointestinal } \\
\text { hemorrhage }\end{array}$ & $2(2.9)$ & $3(4.7)$ & 0.932 & $2(2.9)$ & $3(4.7)$ & 0.932 \\
\hline Pancreatitis & 0 & $\mathrm{I}(\mathrm{I} .6)$ & $0.48 \mathrm{I}$ & 0 & $\mathrm{I}(\mathrm{I} .6)$ & $0.48 \mathrm{I}$ \\
\hline Groin hematoma & $3(4.3)$ & $2(3.1)$ & $>0.999$ & 0 & 0 & $\ldots$ \\
\hline
\end{tabular}

Note: Data are numbers of patients, and data in parentheses are percentages.

Abbreviations: D-TACE-HAIC, drug-eluting bead transarterial chemoembolization combined with hepatic arterial infusion chemotherapy; DEB-TACE, drug-eluting bead transarterial chemoembolization.

The median follow-up duration was 15.4 months (range, 4.5-49.3) for the D-TACE-HAIC group and 12.5 months (range, 3.8-52.1) for the DEB-TACE group. All patients underwent repeated procedures of D-TACE-HAIC or DEB-TACE, with a mean of 2.6 (range, 2-4) and 3.2 (range, 2-5) procedures per patient in the D-TACE-HAIC group and the DEB-TACE group, respectively.

\section{Safety}

Treatment-related AEs are shown in Table 2. No treatment-related mortality occurred. The overall incidence of
AEs was similar between the D-TACE-HAIC group and the DEB-TACE group (any grade: $94.2 \%$ vs $89.1 \%, P=$ 0.282 ; grade $3 / 4: 37.7 \%$ vs $28.1 \%, P=0.242$ ). The following AEs in any grade were more frequent in the D-TACEHAIC group than in the DEB-TACE group: nausea/vomiting $(34.8 \%$ vs $18.8 \%, P=0.038)$, neutropenia $(20.3 \%$ vs $7.8 \%, P=0.040)$, thrombocytopenia $(29.0 \%$ vs $12.5 \%$, $P=0.020)$, and sensory neuropathy $(8.7 \%$ vs $0, P=$ 0.016). However, incidences of all grade $3 / 4$ AEs were not significantly different between the two groups. Treatment schedule was interrupted because of AEs in 
$13.0 \%$ and $7.8 \%$ of patients, respectively, in the D-TACEHAIC group and the DEB-TACE group $(P=0.326)$.

\section{Treatment Response}

Tumor responses of the two groups are shown in Table 3. The ORR and DCR for the D-TACE-HAIC group (Figure 2) were $71.0 \%$ and $94.2 \%$, respectively, which were significantly higher than the $53.1 \%$ and $79.7 \%$ observed in the DEBTACE group ( $P=0.033$ and $P=0.012$, respectively). Subgroup analysis revealed that ORR and DCR in patients with non-smooth tumor margin or macrovascular invasion in the D-TACE-HAIC group were $67.3 \%$ and $94.2 \%$, respectively, which were significantly higher than the $41.7 \%$ and $75.0 \%$ in the DEB-TACE group $(P=0.010$ and $P=0.007$, respectively). However, in patients with smooth tumor margin, ORR and DCR between D-TACE-HAIC group and DEB-TACE group were similar $(82.4 \%$ vs $87.5 \%$, $P>0.999 ; 94.1 \%$ vs $93.8 \%, P>0.999)$.

\section{Overall Survival}

During the follow-up period, 39 of $69(56.5 \%)$ patients in the D-TACE-HAIC group and 51 of $64(79.7 \%)$ patients in the DEB-TACE group died. The 1-, 2-, and 3-year OS rates were $78.5 \%, 36.3 \%$, and $27.7 \%$ (median OS, 19.0 months [95\% CI, 15.0-22.9]), respectively, in the D-TACE-HAIC group and $56.0 \%, 25.7 \%$, and $11.3 \%$ (median OS, 14.0 months [95\% CI, 11.1-16.9]), respectively, in the DEB-TACE group $(P=0.008)$ (Figure 3A). According to uni- and multivariate analysis, DEB-TACE alone (HR, 2.081; 95\% CI, 1.367-3.198; $P=0.001$ ), number of lesions $>3$ (HR, 1.934; 95\% CI, 1.219-3.069;
$P=0.005)$, non-smooth tumor margin (HR, 2.049; 95\% CI, 1.096-3.830; $P=0.025$ ), and macrovascular invasion (HR, 3.471; 95\% CI, 1.902-6.335; $P<0.001$ ) were independent prognostic factors for OS (Table 4).

\section{Progression-Free Survival}

During follow-up, 46 of $69(66.7 \%)$ patients in the D-TACE-HAIC group and 53 of $64(82.8 \%)$ patients in the DEB-TACE group experienced tumor progression. The median PFS in D-TACE-HAIC group vs in DEB-TACE group were 9.3 months (95\% CI, 6.9-11.6) vs 6.3 months (95\% CI, 5.2-7.4), respectively $(P=0.005)$ (Figure 4A). According to uni- and multivariate analysis, DEB-TACE alone (HR, 2.000; 95\% CI, 1.337-2.992; $P=0.001$ ), tumor size $>10 \mathrm{~cm}$ (HR, 1.891; 95\% CI, 1.236-2.894; $P=$ 0.003 ), non-smooth tumor margin (HR, 1.922; 95\% CI, $1.077-3.429 ; P=0.027$ ), and macrovascular invasion (HR, $1.938 ; 95 \%$ CI, 1.126-3.333; $P=0.017$ ) were independent prognostic factors for PFS (Table 5).

\section{Subgroup Analysis of OS and PFS}

In patients with a non-smooth tumor margin or macrovascular invasion, OS and PFS were significantly improved in patients who received D-TACE-HAIC as compared to those who underwent DEB-TACE: the median OS was 20.8 months (95\% CI, 15.7-25.9) vs 13.0 months $(95 \% \mathrm{CI}, 8.4-17.6)(P=0.031)$ and the median PFS was 10.9 months (95\% CI, 9.1-12.7) vs 6.1 months $(95 \% \mathrm{CI}, 4.8-7.4)(P=0.019)$ in patients with non-smooth tumor margin; and the median OS was 15.0 months (95\% CI, 12.4-17.5) vs 11.0 months $(95 \% \mathrm{CI}$,

Table 3 Best Observed Treatment Responses

\begin{tabular}{|c|c|c|c|c|c|c|c|c|c|}
\hline \multirow[b]{2}{*}{$\begin{array}{l}\text { Treatment } \\
\text { Response }\end{array}$} & \multicolumn{3}{|c|}{ Entire Study Population } & \multicolumn{3}{|c|}{ Smooth Tumor Margin } & \multicolumn{3}{|c|}{$\begin{array}{c}\text { Non-Smooth Tumor Margin or } \\
\text { Macrovascular Invasion }\end{array}$} \\
\hline & $\begin{array}{l}\text { D-TACE- } \\
\text { HAIC } \\
\text { Group } \\
(\mathrm{N}=69)\end{array}$ & $\begin{array}{l}\text { DEB- } \\
\text { TACE } \\
\text { Group } \\
(\mathbf{N}=64)\end{array}$ & $P$ value & $\begin{array}{l}\text { D-TACE- } \\
\text { HAIC } \\
\text { Group } \\
(\mathrm{N}=\mid 7)\end{array}$ & $\begin{array}{l}\text { DEB- } \\
\text { TACE } \\
\text { Group } \\
(\mathrm{N}=16)\end{array}$ & $P$ value & $\begin{array}{l}\text { D-TACE- } \\
\text { HAIC } \\
\text { Group (N } \\
=52)\end{array}$ & $\begin{array}{l}\text { DEB- } \\
\text { TACE } \\
\text { Group } \\
(\mathbf{N}=48)\end{array}$ & $P$ value \\
\hline CR & 18 & 11 & $\ldots$ & 9 & 6 & $\ldots$ & 9 & 5 & $\ldots$ \\
\hline PR & 31 & 23 & $\ldots$ & 5 & 8 & $\ldots$ & 26 & 15 & $\ldots$ \\
\hline SD & 16 & 17 & $\ldots$ & 2 & I & $\ldots$ & 14 & 16 & $\ldots$ \\
\hline PD & 4 & 13 & $\ldots$ & I & I & $\ldots$ & 3 & 12 & $\ldots$ \\
\hline ORR, \% & 71.0 & 53.1 & 0.033 & 82.4 & 87.5 & $>0.999$ & 67.3 & 41.7 & 0.010 \\
\hline DCR, \% & 94.2 & 79.7 & 0.012 & 94.1 & 93.8 & $>0.999$ & 94.2 & 75.0 & 0.007 \\
\hline
\end{tabular}

Notes: Except where indicated, data are numbers of patients. Objective response rate $(O R R)=(C R+P R) / N$, and disease control rate $(D C R)=(C R+P R+S D) / N$, where $\mathrm{CR}$ is number of patients with complete response, $\mathrm{PR}$ is number of patients with partial response, $\mathrm{SD}$ is number of patients with stable disease, and $\mathrm{N}$ is total number of patients.

Abbreviation: PD, progressive disease. 

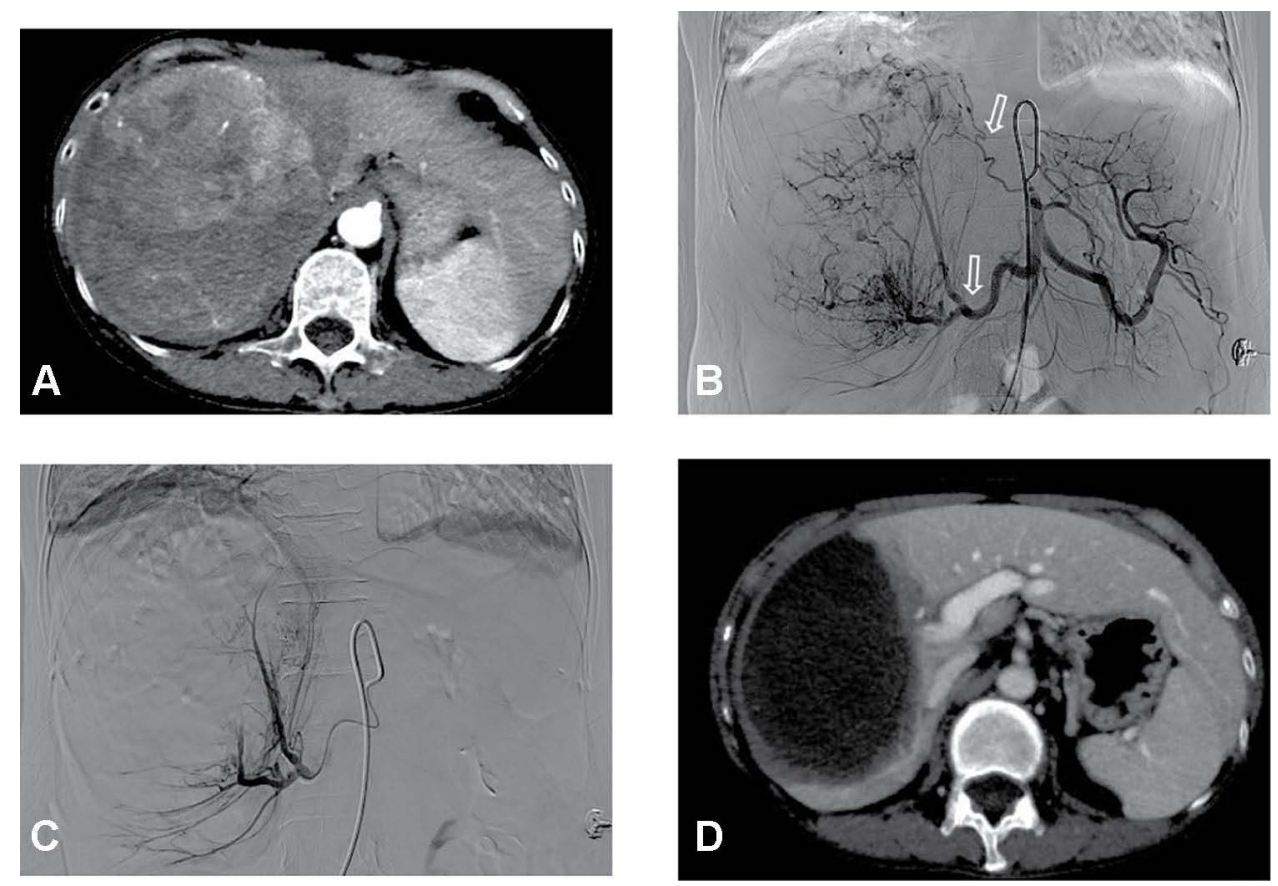

Figure 2 A 69-year-old woman with a huge hepatocellular carcinoma (HCC) who underwent a combination therapy with drug-eluting bead transarterial chemoembolization (DEB-TACE) and hepatic arterial infusion chemotherapy (HAIC). (A) Pretreatment dynamic CT demonstrated a HCC mass of I5.I cm in diameter in the right lobe; (B) digital-subtraction angiography (DSA) before embolization showed the tumor-feeding vessels derived from right hepatic artery and right phrenic artery (arrows), which were then embolized by DEB and Embosphere ${ }^{\circledR}$ beads; $(\mathbf{C})$ after embolization in the first DEB-TACE procedure, DSA showed that the tumor blush remained but significantly reduced, and then the microcatheter was reserved at the right hepatic artery to perform hepatic arterial infusion chemotherapy; (D) dynamic CT images 3 I days after the second procedure of DEB-TACE and HAIC demonstrated shrinkage (I2.5 cm in diameter) and complete necrosis of the targeted tumor and the patency of main trunk and first-order branch of portal vein.

7.7-14.3) $(P=0.015)$ and the median PFS was 8.2 months $(95 \%$ CI, 6.5-9.9) vs 4.7 months $(95 \% \mathrm{CI}$, 3.6-5.8) $(P=0.016)$ in patients with macrovascular invasion. However, in patients with a smooth tumor margin, OS or PFS had no significant difference between D-TACE-HAIC group and DEB-TACE group: the median OS was 37.0 months $(95 \% \mathrm{CI}, 8.9-65.1)$ vs 35.0 months $(95 \% \mathrm{CI}, 14.2-55.8)(P=0.458)$ and the median PFS was 18.0 months $(95 \% \mathrm{CI}, 10.2-25.8)$ vs 13.4 months $(95 \%$ CI, 2.8-24.0) $\quad(P=0.540)$ (Figures 3B-D and 4B-D).

\section{Discussion}

Our study showed that D-TACE-HAIC conferred a significant survival benefit when compared with DEBTACE in patients with large or huge HCC. This may be attributed to a higher ORR and DCR and a longer PFS in patients who underwent D-TACE-HAIC than in those who underwent DEB-TACE. Results of our study in the DEB-TACE group were similar with those previously reported in large or huge HCC undergoing DEB-TACE: the median OS and PFS were 11.5-16.0 months and 6.67.5 months, respectively. ${ }^{12,13,28}$ In our multivariate analyses, combining HAIC was an independent predictor for both better OS and PFS. These results indicated that D-TACE-HAIC had an advantage over DEB-TACE alone for large or huge HCC: DEB-TACE causes substantial tumor ischemic necrosis and sustained drug release, ${ }^{6,7,22}$ while HAIC exposes the residual tumor following embolization to high-concentration chemotherapeutics, and together lead to a better control of tumor.

The difficulty for DEB-TACE treating large or huge HCC mainly lies in the decision of the degree of embolization during per DEB-TACE procedure. In our study, we intended to achieve a complete embolization in 2-3 DEBTACE procedures rather than in the first procedure, which decreased the embolized extent per procedure and embolization-related AEs. In such scenarios, HAIC might play an important role in controlling the residual tumor before complete embolization was achieved. ${ }^{17}$ Besides, combining HAIC did not increase the overall incidence of any grade or grade $3 / 4 \mathrm{AEs}$ on the basis of DEB-TACE.

Large or huge $\mathrm{HCC}$ is often found associated with infiltrative pathological characteristics, ie, micro- or macro-vascular invasion, ${ }^{19,29,30}$ which is usually presented as a non-smooth tumor margin or macrovascular invasion 

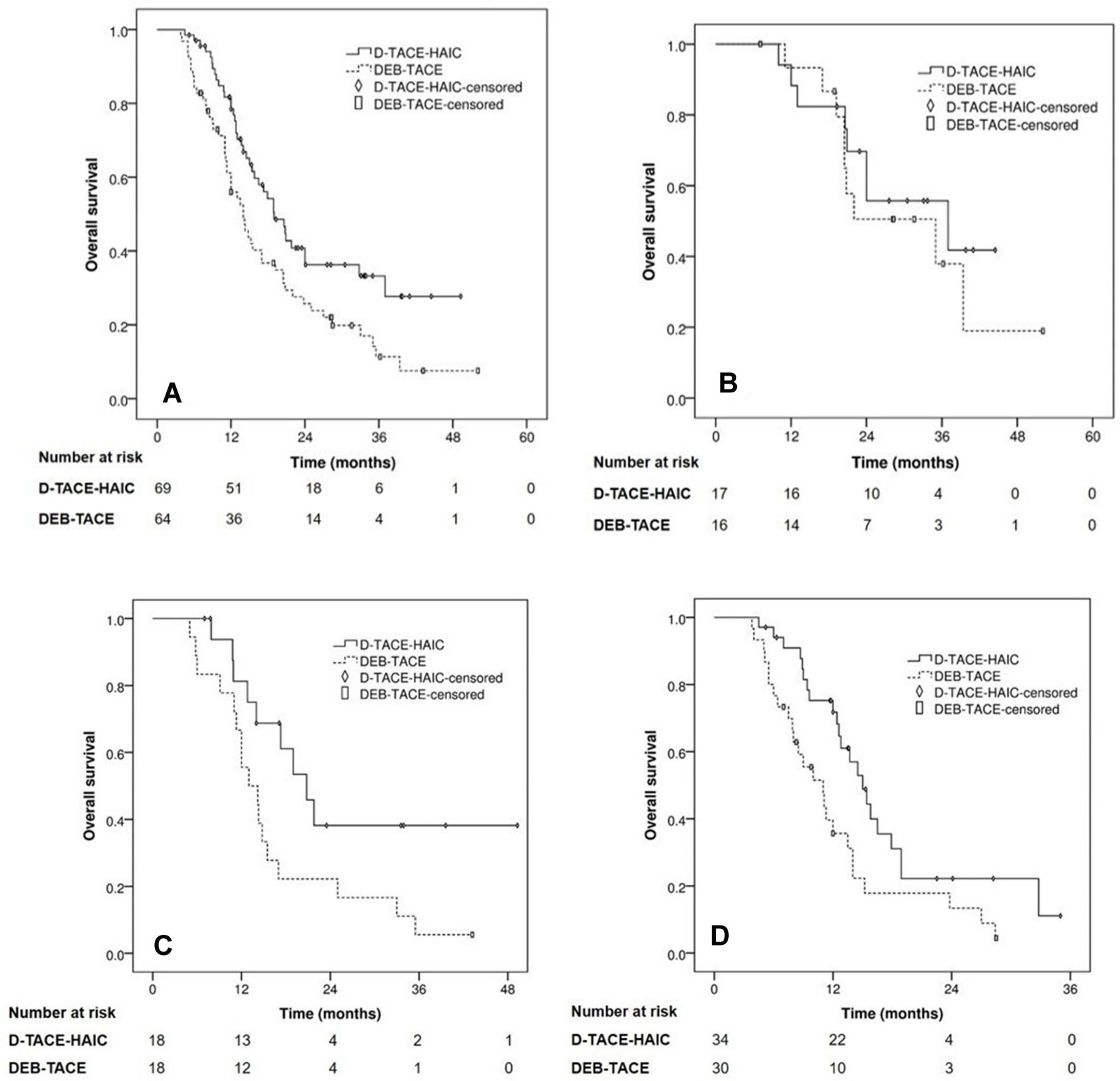

Figure 3 Kaplan-Meier curves of overall survival for patients with large or huge hepatocellular carcinoma who underwent drug-eluting bead transarterial chemoembolization (DEB-TACE) plus hepatic arterial infusion chemotherapy (D-TACE-HAIC) or DEB-TACE. $($ A) Entire study population $(D-T A C E-H A I C$ group: $n=69$, median survival = 19.0 months; DEB-TACE group: $\mathrm{n}=64$, median survival $=14.0$ months; $P=0.008)$. $(B)$ Patients with smooth tumor margin $(D-T A C E-H A I C$ group: $n=I 7$, median survival $=$ 37.0 months; DEB-TACE group: $\mathrm{n}=16$, median survival $=35.0$ months; $P=0.458)$. (C) Patients with non-smooth tumor margin $(\mathrm{D}-\mathrm{TACE}-\mathrm{HAIC}$ group: $\mathrm{n}=18$, median survival $=20.8$ months; DEB-TACE group: $n=18$, median survival $=13.0$ months; $P=0.031)$. (D) Patients with macrovascular invasion $(D-T A C E-H A I C$ group: $n=34$, median survival $=15.0$ months; DEB-TACE group: $\mathrm{n}=30$, median survival $=11.0$ months; $P=0.015)$.

at imaging. ${ }^{24,31}$ In our study, huge HCC had a higher proportion of non-smooth tumor margin or macrovascular invasion than large $\mathrm{HCC}(85.7 \%$ vs $65.7 \%)$. In the previous studies, outcomes were poor in HCC patients with non-smooth tumor margin or macrovascular invasion who underwent DEB-TACE. ${ }^{25,32}$ In our multivariate analyses, non-smooth margin and macrovascular invasion were independent risk factors for both poor OS and PFS, while tumor $>10 \mathrm{~cm}$ independently predicted poor PFS.

Importantly, in patients with a non-smooth tumor margin or macrovascular invasion, combining HAIC significantly improved the ORR and DCR, and the OS and PFS were also increased significantly compared with the DEB-TACE group. Intrahepatic metastasis occurs more 
Table 4 Analyses of Prognostic Factors for Overall Survival

\begin{tabular}{|c|c|c|c|c|}
\hline \multirow[t]{2}{*}{ Factor } & \multicolumn{2}{|c|}{ Univariate Analysis } & \multicolumn{2}{|c|}{ Multivariate Analysis } \\
\hline & HR (95\% Cl) & $P$ value & HR (95\% Cl) & $P$ value \\
\hline DEB-TACE alone & 1.737 (I.144-2.637) & 0.010 & 2.091 (1.367-3.198) & 0.001 \\
\hline Male & $0.902(0.479-1.698)$ & 0.749 & & \\
\hline$>60$ years & $0.975(0.613-1.548)$ & 0.913 & & \\
\hline BCLC stage $B$ & $3.069(0.943-9.987)$ & 0.063 & $\ldots$ & 0.533 \\
\hline BCLC stage $C$ & $6.268(1.94 I-20.243)$ & 0.002 & $\ldots$ & 0.615 \\
\hline ECOG score $=1$ & 1.745 (1.069-2.848) & 0.026 & $\ldots$ & 0.821 \\
\hline Positive for HbsAg & $1.237(0.566-2.705)$ & 0.594 & & \\
\hline Tumor size $>10 \mathrm{~cm}$ & $1.706(1.125-2.586)$ & 0.012 & $\ldots$ & 0.180 \\
\hline$>3$ lesions & $2.363(I .52 I-3.67 \mid)$ & $<0.001$ & $1.934(1.219-3.069)$ & 0.005 \\
\hline Second- or lower-order portal vein branch invasion & $2.083(1.198-3.621)$ & 0.009 & $\ldots$ & 0.530 \\
\hline First-order portal vein branch invasion & $3.023(1.831-4.990)$ & $<0.001$ & $\ldots$ & 0.530 \\
\hline Non-smooth tumor margin & $2.280(1.234-4.216)$ & 0.009 & $2.049(1.096-3.830)$ & 0.025 \\
\hline Macrovascular invasion & $3.907(2.195-6.953)$ & $<0.001$ & $3.471(1.902-6.335)$ & $<0.001$ \\
\hline$\alpha$-Fetoprotein $>400 \mathrm{ng} / \mathrm{mL}$ & $1.057(0.694-1.611)$ & 0.797 & & \\
\hline Total bilirubin $>22$ umol/L & $1.291(0.803-2.074)$ & 0.292 & & \\
\hline Albumin $<35 \mathrm{~g} / \mathrm{dL}$ & $1.223(0.782-1.913)$ & 0.377 & & \\
\hline Platelet $<100 \times 10^{9} / \mathrm{L}$ & $1.366(0.725-2.573)$ & 0.334 & & \\
\hline Sorafenib/lenvatinib (vs locoregional therapy) in post treatment & $1.957(1.028-3.724)$ & $0.04 I$ & $\ldots$ & 0.651 \\
\hline $\begin{array}{l}\text { Combination of sorafenib/lenvatinib and locoregional therapy (vs } \\
\text { locoregional therapy) in post treatment }\end{array}$ & $1.542(0.818-2.908)$ & 0.181 & $\ldots$ & 0.997 \\
\hline Best supportive care (vs locoregional therapy) in post treatment & $1.998(0.705-5.666)$ & 0.193 & $\ldots$ & 0.197 \\
\hline
\end{tabular}

Note: The uni- and multivariate analyses were performed using Cox proportional hazard regression model.

Abbreviations: HR, Hazard Ratio; Cl, confidence interval; BCLC, Barcelona Clinic Liver Cancer; ECOG, Eastern Cooperative Oncology Group; DEB-TACE, drug-eluting bead transarterial chemoembolization; $\mathrm{HBsAg}$, hepatitis $\mathrm{B}$ surface antigen.

often in HCC patients with a non-smooth margin or macrovascular invasion ${ }^{33}$ and HAIC was reported to effectively decrease the occurrence of intrahepatic metastasis in these patients. ${ }^{34}$ This might be an important reason why D-TACE-HAIC could improve the outcomes in patients with a non-smooth tumor margin or macrovascular invasion. By contrast, in HCC with a smooth tumor margin, D-TACE-HAIC and DEB-TACE led to a similar median OS (37.0 vs 35.0 months), consistent with the median OS of 33.4-35.6 months for large noninfiltrative HCC undergoing DEB-TACE in previous studies. ${ }^{10,11}$ In our study, patients with a smooth tumor margin had a high proportion (72.7\% [24/33]) of cases with tumor size of $5.1-10 \mathrm{~cm}$, and a similar ORR was achieved in the two groups $(82.4 \%$ vs $87.5 \%)$. These results indicated that, for patients with a smooth tumor margin and tumor size of 5.1-10 cm, DEB-TACE alone might achieve an excellent efficacy and combining HAIC may not benefit. Further study is necessary to confirm this finding.

FOLFOX-based HAIC alone is also adopted in treating large or huge HCC and recent studies reported a median
OS and PFS of 13.9 and 5.9 months. ${ }^{20,21}$ These results seem worse than the outcomes of the D-TACE-HAIC group in our study (19.0 and 9.3 months). Two studies focused on FOLFOX-based HAIC combined with c-$\mathrm{TACE}^{35}$ or bland embolization (TAE) ${ }^{36}$ in intermediateadvanced stage HCC. The median PFS in these studies for the entire treatment group and subgroup of macrovascular invasion were 7.9-8.0 months and 4.2-6.5 months, respectively. It seems that our D-TACE-HAIC group led to better PFS (9.3 and 8.2 months) than c-TACE+HAIC or TAE +HAIC, even though patients in our study had a greater tumor burden, which might be because DEB-TACE offered superior anticancer activity as compared to c-TACE or TAE. ${ }^{8,37}$ Besides, compared with c-TACE, DEB-TACE causes substantially lower level of chemotherapeutics in the systemic circulation ${ }^{6,7}$ and provides a standardized and repeatable procedure not available with c-TACE. ${ }^{16}$ Thus, D-TACE-HAIC should be a more appropriate treatment for large or huge HCC.

About half of the patients in our study had advanced HCC. At the time when these patients received their primary treatment, tyrosine kinase inhibitors (TKI) sorafenib 

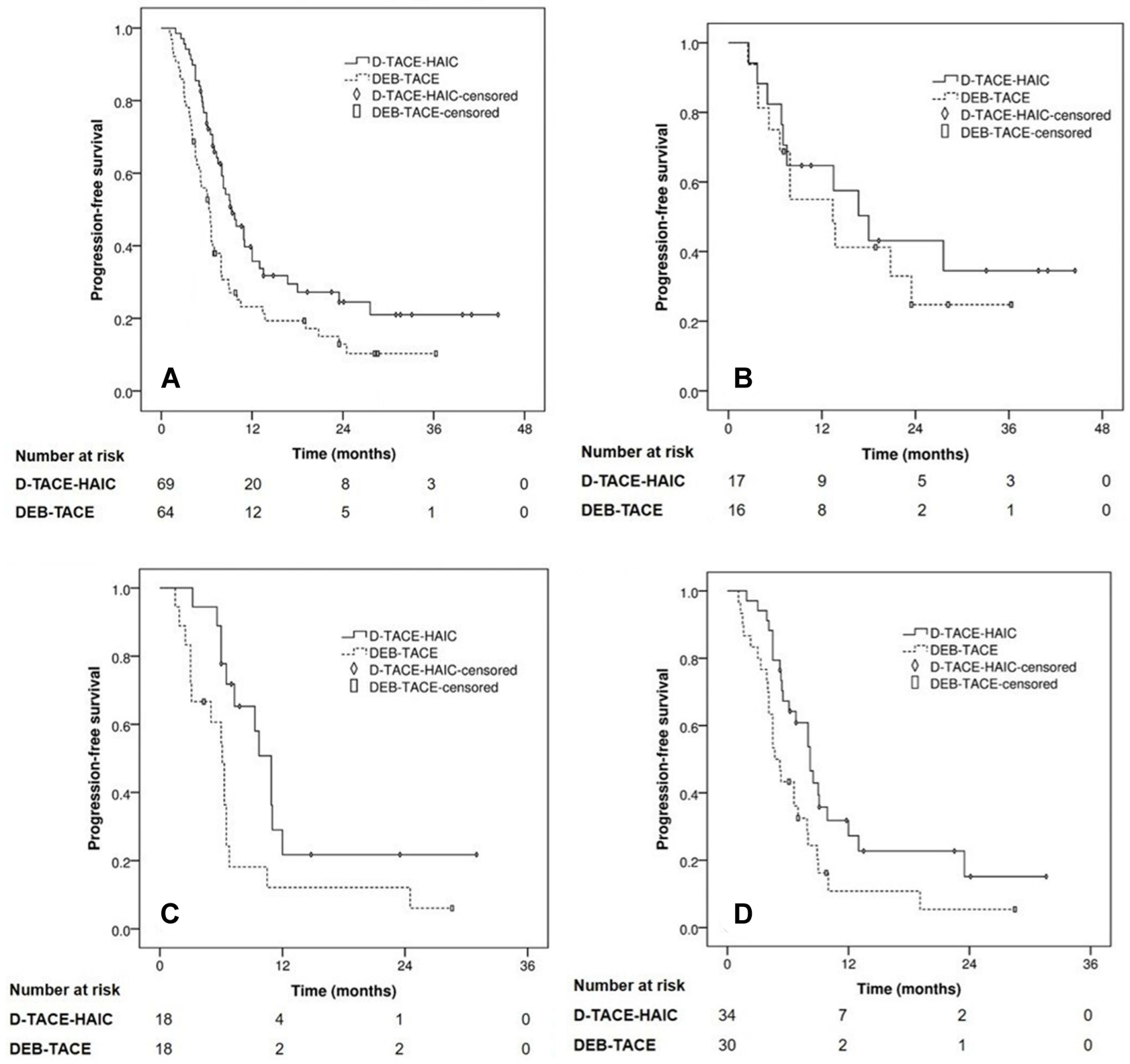

Figure 4 Kaplan-Meier curves of progression-free survival (PFS) for patients with large or huge hepatocellular carcinoma who underwent drug-eluting bead transarterial chemoembolization (DEB-TACE) plus hepatic arterial infusion chemotherapy (D-TACE-HAIC) or DEB-TACE. (A) Entire study population (D-TACE-HAIC group: $n=69$, median PFS $=9.3$ months; DEB-TACE group: $n=64$, median PFS $=6.3$ months; $P=0.005$ ). (B) Patients with smooth tumor margin (D-TACE-HAIC group: $n=17$, median PFS = 18.0 months; DEB-TACE group: $n=16$, median PFS = 13.4 months; $P=0.540)$. (C) Patients with non-smooth tumor margin (D-TACE-HAIC group: $n=18$, median PFS $=10.9$ months; DEB-TACE group: $\mathrm{n}=18$, median PFS $=6.1$ months; $P=0.019)$. $(\mathrm{D})$ Patients with macrovascular invasion (D-TACE-HAIC group: $\mathrm{n}=34$, median PFS $=8.2$ months; DEB-TACE group: $\mathrm{n}=30$, median PFS $=4.7$ months; $P=0.016$ ).

or lenvatinib were standard first-line treatment of advanced $\mathrm{HCC}$, and other TKIs (eg, regorafenib) and immune checkpoint inhibitors (ICI) (eg, PD-1 inhibitors) were post-line treatments. However, the efficacy of sorafenib or lenvatinib alone for HCC was moderate, ${ }^{38,39}$ especially in the large or locally advanced HCC. In the recent study, for large locally advanced HCC without extrahepatic metastasis, locoregional treatment HAIC had an better efficacy than sorafenib. ${ }^{20}$ Moreover, it was reported that lenvatinib treatment alone brought tumorrelated hemorrhages in large $\mathrm{HCC}^{40}$ Actually, the TKIs are usually used in combination with local treatment in large or locally advanced HCC. ${ }^{41-43}$ In our study, for these large and locally advanced HCC with no extrahepatic metastasis, the primary treatment with locoregional treatment should be an appropriate treatment option. The 
Table 5 Analyses of Prognostic Factors for Progression-Free Survival

\begin{tabular}{|c|c|c|c|c|}
\hline \multirow[t]{2}{*}{ Factor } & \multicolumn{2}{|c|}{ Univariate Analysis } & \multicolumn{2}{|c|}{ Multivariate Analysis } \\
\hline & HR (95\% Cl) & $P$ value & HR (95\% Cl) & $P$ value \\
\hline DEB-TACE alone & 1.748 (I.176-2.599) & 0.006 & 2.000 (1.337-2.992) & 0.001 \\
\hline Male & $0.748(0.408-1.369)$ & 0.346 & & \\
\hline$>60$ years & $0.693(0.437-1.099)$ & 0.119 & & \\
\hline $\mathrm{BCLC}$ stage $\mathrm{B}$ & 3.067 (1.208-7.785) & 0.018 & $\ldots$ & 0.129 \\
\hline BCLC stage $C$ & 3.463 (1.375-8.722) & 0.008 & $\ldots$ & 0.290 \\
\hline ECOG score $=1$ & I.I52 (0.697-I.904) & 0.582 & & \\
\hline Positive for HbsAg & $1.384(0.640-2.997)$ & 0.409 & & \\
\hline Tumor size $>10 \mathrm{~cm}$ & $1.979(1.32 \mathrm{I}-2.965)$ & 0.001 & 1.891 (1.236-2.894) & 0.003 \\
\hline$>3$ lesions & $1.662(1.107-2.495)$ & 0.014 & $\ldots$ & 0.455 \\
\hline Second- or lower-order portal vein branch invasion & $1.364(0.813-2.290)$ & 0.240 & $\ldots$ & 0.597 \\
\hline First-order portal vein branch invasion & $1.736(1.092-2.760)$ & 0.020 & $\ldots$ & 0.597 \\
\hline Non-smooth tumor margin & $1.940(1.095-3.439)$ & 0.023 & $1.922(1.077-3.429)$ & 0.027 \\
\hline Macrovascular invasion & $2.182(1.296-3.673)$ & 0.003 & $1.938(1.126-3.333)$ & 0.017 \\
\hline$\alpha$-Fetoprotein $>400 \mathrm{ng} / \mathrm{mL}$ & $1.064(0.713-1.588)$ & 0.761 & & \\
\hline Total bilirubin $>22 \mu \mathrm{mol} / \mathrm{L}$ & $1.270(0.806-1.999)$ & 0.303 & & \\
\hline Albumin $<35 \mathrm{~g} / \mathrm{dL}$ & $0.939(0.612-1.442)$ & 0.774 & & \\
\hline Platelet $<100 \times 10^{9} / \mathrm{L}$ & $1.180(0.629-2.212)$ & 0.606 & & \\
\hline
\end{tabular}

Note: The uni- and multivariate analyses were performed using Cox proportional hazard regression model.

Abbreviations: HR, Hazard Ratio; Cl, confidence interval; BCLC, Barcelona Clinic Liver Cancer; ECOG, Eastern Cooperative Oncology Group; DEB-TACE, drug-eluting bead transarterial chemoembolization; $\mathrm{HBsAg}$, hepatitis $\mathrm{B}$ surface antigen.

patients with locally advanced large HCC were treated by TKIs alone or in combination with ICIs or local treatment when the primary treatment failed.

This study has several limitations. First, as a retrospective study, the comparison of D-TACE-HAIC and DEB-TACE might be subject to selection bias, and no matched pair analysis between the two groups was performed because of a relatively small sample of study population. However, we conducted multivariate analyses and subgroup analyses to make a correction for confounding factors. Second, the efficacy of D-TACE-HAIC for HCC $>10 \mathrm{~cm}$ with a smooth tumor margin remained unclear. Although the subgroup analysis of patients with a smooth tumor margin showed similar OS between D-TACE-HAIC and DEB-TACE group, among these patients only $27 \%(9 / 33)$ had $\mathrm{HCC}>10 \mathrm{~cm}$. Third, we did not conduct direct comparison between D-TACE-HAIC and HAIC. Instead, we compared the outcomes of D-TACEHAIC group in our study with those previously reported in large or huge HCC undergoing HAIC alone and found that there might be better outcomes for D-TACE-HAIC, which needed conformation by further studies.

In conclusion, D-TACE-HAIC was tolerable and yielded promising outcomes in patients with large or huge HCC. These patients appeared to benefit from D-TACE-HAIC, and have better treatment responses, PFS, and OS, in comparison to DEB-TACE, especially in those who had a non-smooth tumor margin or macrovascular invasion.

\section{Funding}

This study has received funding by the National Natural Science Foundation of China (Grant No. 82001930, 81873920), the Science and Technology Program of Guangzhou, China (Grant No.202002030135), and HighLevel University Clinical Research Promotion Program of Guangzhou Medical University (No. B185004019).

\section{Disclosure}

The authors report no conflicts of interest in this work.

\section{References}

1. Galle PR, Forner A, Llovet JM, et al; European Association for the Study of the Liver. EASL Clinical Practice Guidelines: management of hepatocellular carcinoma. $J$ Hepatol. 2018;69(1):182-236. doi:10.1016/j.jhep.2018.03.019

2. Li B, Qiu J, Zheng Y, et al. Conversion to resectability using transarterial chemoembolization combined with hepatic arterial infusion chemotherapy for initially unresectable hepatocellular carcinoma. Ann Surg. 2021;2:e057. doi:10.1097/AS9.0000000000000057

3. Song MJ, Park CH, Kim JD, et al. Drug-eluting bead loaded with doxorubicin versus conventional Lipiodol-based transarterial chemoembolization in the treatment of hepatocellular carcinoma: a case-control study of Asian patients. Eur J Gastroenterol Hepatol. 2011;23(6):521-527. doi:10.1097/MEG.0b013e328346d505 
4. Xue T, Le F, Chen R, et al. Transarterial chemoembolization for huge hepatocellular carcinoma with diameter over ten centimeters: a large cohort study. Med Oncol. 2015;32(3):64. doi:10.1007/s12032-0150504-3

5. Huang YH, Wu JC, Chen SC, et al. Survival benefit of transcatheter arterial chemoembolization in patients with hepatocellular carcinoma larger than $10 \mathrm{~cm}$ in diameter. Aliment Pharmacol Ther. 2006;23 (1):129-135. doi:10.1111/j.1365-2036.2006.02704.x

6. Hagan A, Caine M, Press C, et al. Predicting pharmacokinetic behaviour of drug release from drug-eluting embolization beads using in vitro elution methods. Eur J Pharm Sci. 2019;136:104943. doi:10.1016/j.ejps.2019.05.021

7. Zhang S, Huang C, Li Z, et al. Comparison of pharmacokinetics and drug release in tissues after transarterial chemoembolization with doxorubicin using diverse lipiodol emulsions and CalliSpheres Beads in rabbit livers. Drug Deliv. 2017;24(1):1011-1017. doi:10.1080/10717544.2017.1344336

8. Song MJ, Chun HJ, Song DS, et al. Comparative study between doxorubicin-eluting beads and conventional transarterial chemoembolization for treatment of hepatocellular carcinoma. J Hepatol. 2012;57(6):1244-1250. doi:10.1016/j.jhep.2012.07.017

9. Zhang ZS, Li HZ, Ma C, Xiao YD. Conventional versus drug-eluting beads chemoembolization for infiltrative hepatocellular carcinoma: a comparison of efficacy and safety. BMC Cancer. 2019;19(1):1162. doi:10.1186/s12885-019-6386-6

10. Ou MC, Liu YS, Chuang MT, et al. Time-to-progression following conventional compared with drug-eluting-bead transcatheter arterial chemoembolisation in patients with large hepatocellular carcinoma. Clin Radiol. 2019;74(4):295-300. doi:10.1016/j.crad.2018.12.008

11. Lee YK, Jung KS, Kim DY, et al. Conventional versus drug-eluting beads chemoembolization for hepatocellular carcinoma: emphasis on the impact of tumor size. $J$ Gastroenterol Hepatol. 2017;32 (2):487-496. doi:10.1111/jgh.13501

12. Zhang X, Lin X, Qiu H, et al. An investigation of efficacy, safety, and prognostic factors of drug-eluting beads-transarterial chemoembolization operation with CalliSpheres ${ }^{\circledR}$ Microspheres in treating Chinese hepatocellular carcinoma patients. J Clin Lab Anal. 2019;33(8): e22975. doi:10.1002/jcla.22975

13. Gomes AS, Monteleone PA, Sayre JW, et al. Comparison of triple-drug Transcatheter Arterial Chemoembolization (TACE) with single-drug TACE using doxorubicin-eluting beads: long-term survival in 313 patients. AJR Am J Roentgenol. 2017;209(4):722-732. doi:10.2214/AJR.17.18219

14. Paul SB, Gamanagatti S, Sreenivas V, et al. Trans-arterial chemoembolization (TACE) in patients with unresectable Hepatocellular carcinoma: experience from a tertiary care centre in India. Indian $J$ Radiol Imaging. 2011;21(2):113-120. doi:10.4103/09713026.82294

15. Hiraoka A, Horiike N, Yamashita Y, et al. Risk factors for death in 224 cases of hepatocellular carcinoma after transcatheter arterial chemoembolization. Hepatogastroenterology. 2009;56(89):213-217. doi:10.1016/S0168-583X(98)00414-5

16. Lencioni R, de Baere T, Burrel M, et al. Transcatheter treatment of hepatocellular carcinoma with Doxorubicin-loaded DC Bead (DEBDOX): technical recommendations. Cardiovasc Intervent Radiol. 2012;35(5):980-985. doi:10.1007/s00270-011-0287-7

17. Nakano MM, Yamamoto A, Nishida N, et al. Risk factors for local recurrence of hepatocellular carcinoma after transcatheter arterial chemoembolization with drug-eluting beads (DEB-TACE). Jpn J Radiol. 2019;37(7):543-548. doi:10.1007/s11604-019-00840-4

18. Yoon HM, Kim JH, Kim EJ, et al. Modified cisplatin-based transcatheter arterial chemoembolization for large hepatocellular carcinoma: multivariate analysis of predictive factors for tumor response and survival in a 163-patient cohort. J Vasc Interv Radiol. 2013;24 (11):1639-1646. doi:10.1016/j.jvir.2013.06.017
19. Zheng J, Chakraborty J, Chapman WC, et al. Preoperative prediction of microvascular invasion in hepatocellular carcinoma using quantitative image analysis. J Am Coll Surg. 2017;225(6):778-788.e1. doi:10.1016/j.jamcollsurg.2017.09.003

20. Lyu N, Zhao M. Hepatic arterial infusion chemotherapy of oxaliplatin plus fluorouracil versus sorafenib in advanced hepatocellular carcinoma: a biomolecular exploratory, randomized, Phase 3 trial (The FOHAIC-1 study). $J$ Clin Oncol. 2021;39(15_suppl):4007. doi:10.1200/JCO.2021.39.15_suppl.4007

21. He MK, Le Y, Li QJ, et al. Hepatic artery infusion chemotherapy using mFOLFOX versus transarterial chemoembolization for massive unresectable hepatocellular carcinoma: a prospective non-randomized study. Chin J Cancer. 2017;36(1):83. doi:10.1186/s40880-017-0251-2

22. Namur J, Citron SJ, Sellers MT, et al. Embolization of hepatocellular carcinoma with drug-eluting beads: doxorubicin tissue concentration and distribution in patient liver explants. J Hepatol. 2011;55 (6):1332-1338. doi:10.1186/s40880-017-0251-2

23. Marrero JA, Kulik LM, Sirlin CB, et al. Diagnosis, staging, and management of hepatocellular carcinoma: 2018 practice guidance by the American Association for the Study of Liver Diseases. Hepatology. 2018;68(2):723-750. doi:10.1002/hep.29913

24. Renzulli M, Brocchi S, Cucchetti A, et al. Can current preoperative imaging be used to detect microvascular invasion of hepatocellular carcinoma? Radiology. 2016;279(2):432-442. doi:10.1148/ radiol.2015150998

25. Fronda M, Doriguzzi Breatta A, Gatti M, et al. Quantitative assessment of HCC wash-out on CT is a predictor of early complete response to TACE. Eur Radiol. 2021;31(9):6578-6588. doi:10.1007/s00330-021-07792-2

26. National Cancer Institute. Common terminology criteria for adverse events, version 5.0; 2017. Available from: https://ctep.cancer.gov/ protocolDevelopment/electronic applications/ctc.htm\#ctc 50 . Accessed December 16, 2020.

27. Lencioni R, Llovet JM. Modified RECIST (mRECIST) assessment for hepatocellular carcinoma. Semin Liver Dis. 2010;30(1):52-60. doi:10.1055/s-0030-1247132

28. Zhou Y. Drug-eluting bead transarterial chemoembolization is efficient and well-tolerated in treating elderly Chinese hepatocellular carcinoma patients. Int J Clin Exp Pathol. 2018;11(10):4867-4878.

29. Vernuccio F, Porrello G, Cannella R, et al. Benign and malignant mimickers of infiltrative hepatocellular carcinoma: tips and tricks for differential diagnosis on CT and MRI. Clin Imaging. 2021;70:33-45. doi:10.1016/j.clinimag.2020.10.011

30. Hong SB, Choi SH, Kim SY, et al. MRI features for predicting microvascular invasion of hepatocellular carcinoma: a systematic review and meta-analysis. Liver Cancer. 2021;10(2):94-106. doi:10.1159/000513704

31. Chou CT, Chen RC, Lin WC, Ko CJ, Chen CB, Chen YL. Prediction of microvascular invasion of hepatocellular carcinoma: preoperative CT and histopathologic correlation. AJR Am J Roentgenol. 2014;203 (3):W253-W259. doi:10.2214/AJR.13.10595

32. Sellers MT, Huggins S, Kegley K, et al. Multivariate analysis of prognostic factors for survival following doxorubicin-eluting bead transarterial chemoembolization for hepatocellular carcinoma. J Vasc Interv Radiol. 2013;24(5):647-654. doi:10.1016/j. jvir.2012.12.003

33. Imamura H, Matsuyama $Y$, Tanaka E, et al. Risk factors contributing to early and late phase intrahepatic recurrence of hepatocellular carcinoma after hepatectomy. J Hepatol. 2003;38(2):200-207. doi:10.1016/s0168-8278(02)00360-4

34. Kawabe N, Hashimoto S, Nakano T, Nakaoka K, Fukui A, Yoshioka K. Transcatheter arterial infusion chemotherapy with cisplatin in combination with transcatheter arterial chemoembolization decreases intrahepatic distant recurrence of unresectable hepatocellular carcinoma. JGH Open. 2021;5(6):705-711. doi:10.1002/ jgh3.12573 
35. Gao S, Zhang PJ, Guo JH, et al. Chemoembolization alone vs combined chemoembolization and hepatic arterial infusion chemotherapy in inoperable hepatocellular carcinoma patients. World J Gastroenterol. 2015;21 (36):10443-10452. doi:10.3748/wjg.v21.i36.10443

36. Guo W, Gao J, Zhuang W, Wu Z, Li B, Chen S. Efficacy and safety of hepatic arterial infusion chemotherapy combined with transarterial embolization for unresectable hepatocellular carcinoma: a propensity score-matching cohort study. JGH Open. 2019;4(3):477-483. doi:10.1002/jgh3.12285

37. Malagari K, Pomoni M, Kelekis A, et al. Prospective randomized comparison of chemoembolization with doxorubicin-eluting beads and bland embolization with BeadBlock for hepatocellular carcinoma. Cardiovasc Intervent Radiol. 2010;33(3):541-551. doi:10.1007/s00270-009-9750-0

38. Kudo M, Finn RS, Qin S, et al. Lenvatinib versus sorafenib in first-line treatment of patients with unresectable hepatocellular carcinoma: a randomised phase 3 non-inferiority trial. Lancet. 2018;391 (10126):1163-1173. doi:10.1016/S0140-6736(18)30207-1

39. Llovet JM, Ricci S, Mazzaferro V, et al.; SHARP Investigators Study Group. Sorafenib in advanced hepatocellular carcinoma. $N$ Engl $J$ Med. 2008;359(4):378-390. doi:10.1056/NEJMoa0708857
40. Uchida-Kobayashi S, Kageyama K, Yamamoto A, et al. Lenvatinibinduced tumor-related hemorrhages in patients with large hepatocellular carcinomas. Oncology. 2021;99(3):186-191. doi:10.1159/ 000510911

41. He M, Li Q, Zou R, et al. Sorafenib plus hepatic arterial infusion of oxaliplatin, fluorouracil, and leucovorin vs sorafenib alone for hepatocellular carcinoma with portal vein invasion: a randomized clinical trial. JAMA Oncol. 2019;5(7):953-960. doi:10.1001/ jamaoncol.2019.0250

42. Zhu K, Huang J, Lai L, et al. Medium or large hepatocellular carcinoma: sorafenib combined with transarterial chemoembolization and radiofrequency ablation. Radiology. 2018;288(1):300-307. doi:10.1148/radiol.2018172028

43. Ding X, Sun W, Chen J, et al. Percutaneous radiofrequency ablation combined with transarterial chemoembolization plus sorafenib for large hepatocellular carcinoma invading the portal venous system: a prospective randomized study. Front Oncol. 2020;10:578633. doi: $10.3389 /$ fonc. 2020.578633
Journal of Hepatocellular Carcinoma

\section{Publish your work in this journal}

The Journal of Hepatocellular Carcinoma is an international, peerreviewed, open access journal that offers a platform for the dissemination and study of clinical, translational and basic research findings in this rapidly developing field. Development in areas including, but not limited to, epidemiology, vaccination, hepatitis therapy, pathology

\section{Dovepress}

and molecular tumor classification and prognostication are al considered for publication. The manuscript management system is completely online and includes a very quick and fair peer-review system, which is all easy to use. Visit http://www.dovepress.com/ testimonials.php to read real quotes from published authors. 